\title{
Continued Complete Hematologic Response
}

National Cancer Institute

\section{Source}

National Cancer Institute. Continued Complete Hematologic Response. NCI Thesaurus.

Code C129648.

Durable or long-term complete hematologic response. Time frames that define durability of complete hematologic response vary for different hematologic malignancies. 